\title{
Short communication: Validation of a point-of-care glucometer for use in dairy cows
}

\author{
J. A. M. Wittrock, ${ }^{1}$ T. F. Duffield, and S. J. LeBlanc \\ Department of Population Medicine, Ontario Veterinary College, University of Guelph, Guelph, Ontario, N1G 2W1, Canada
}

\begin{abstract}
The purpose of this study was to evaluate the diagnostic performance of a hand-held electronic glucometer (Precision Xtra; Abbott Diabetes Care Inc., Mississauga, ON, Canada) for cow-side use in dairy cattle. This device has been validated for measuring blood concentrations of $\beta$-hydroxybutyrate in dairy cows. This study was designed to assess the accuracy of whole-blood glucose measurements from the glucose meter relative to a reference chemical analyzer in a diagnostic laboratory. Duplicate samples were taken from the same cows at the same time, into blood tubes with either the glycolysis-inhibiting preservative sodium fluoride $(\mathrm{NaF})$ or without preservative. Glucometer readings were taken on whole blood with no preservative, and laboratory measurements were conducted on serum preserved with NaF. Blood samples were collected from cows between 3 wk before and 5 wk after calving, including during a glucose tolerance test conducted 1 wk before expected calving. Passing-Bablok and Bland-Altman data analyses were used to evaluate the performance of the glucometer relative to the laboratory results. A strong correlation was observed in 709 samples from 81 cows between the hand-held meter and serum from samples preserved with $\mathrm{NaF}\left(\mathrm{R}^{2}=0.95\right)$. Overall, $96 \%$ of measurements with the glucometer fell within the $95 \%$ confidence limits of analysis in the laboratory, although at higher-than-physiologic glucose concentrations $(>5.2 \mathrm{mmol} / \mathrm{L})$ the glucometer tended to overestimate. The hand-held glucometer appears suitable for rapid measurement of glucose under field conditions in dairy cattle.
\end{abstract}

Key words: glucometer, insulin resistance, glucose tolerance test, peripartum

\section{Short Communication}

Beginning in late gestation and continuing into early lactation, dairy cows are insulin resistant as glucose

\footnotetext{
Received December 28, 2012 .

Accepted April 2, 2013.

${ }^{1}$ Corresponding author: julie.wittrock@gmail.com
}

produced is partitioned primarily to the mammary gland (Hayirli, 2006). Changes in the responsiveness (hormone release in response to stimuli) or sensitivity (tissue responsiveness to hormone), or both, may result in insulin resistance (IR; Kahn, 1978). Due to the difficulty in discerning the relative importance of responsiveness and sensitivity in most cases, particularly in the transition period when different tissues are involved, IR is used as a general term for the phenomenon (Schoenberg and Overton, 2010). Glucose tolerance tests (GTT) can be used to measure IR (Lozner et al., 1941; Hayirli, 2006). This procedure consists of an intravenous bolus infusion of $50 \%$ glucose and monitoring of serum concentrations of glucose and insulin at regular intervals. A simplified GTT has been suggested by Matteo et al. (2009), whereby the ratio of glucose concentration $80 \mathrm{~min}$ after glucose infusion to that before infusion are compared, with a ratio of 1.05 proposed as an interpretive cut point. The same research group (Riuzzi et al., 2012) reported an association between the GTT ratio and postpartum BHBA concentration. However, the utility of the simplified GTT in general, and the predictive value of particular cut points for subsequent health outcomes have not been validated. Additionally, recent data indicate that glucose concentration at 3 DIM may be a useful predictor of pregnancy at first AI (Garverick et al., 2013). The ability to rapidly and inexpensively measure blood glucose concentrations cowside would be useful in assessing the metabolic status of dairy cows.

Several studies have reported the use of a variety of hand-held human electronic glucometers in dairy cattle, but few report data on validation or test characteristics (Leury et al., 2003; Fall et al., 2008; Galvão et al., 2010). One glucose and ketone hand-held meter (Precision Xtra; Abbott Diabetes Care Inc., Mississauga, ON, Canada) has been validated for diagnosis of subclinical ketosis in dairy cows, with a Pearson correlation coefficient of 0.95 (Iwersen et al., 2009). The ability of this device to accurately measure glucose in dairy cows was briefly evaluated with a reported coefficient of determination value of 0.56 (Oetzel and McGuirk, 2008).

The purpose of this study was to evaluate the diagnostic performance of a hand-held glucometer (Pre- 
cision Xtra) for cowside use in dairy cattle. Specific objectives were to (1) assess the accuracy of wholeblood glucose measurements from the Precision Xtra glucometer relative to the gold standard of a reference chemical analyzer in a diagnostic laboratory and (2) assess the suitability of the glucometer to classify cows as insulin resistant with a GTT.

A hand-held electronic glucose measuring system (Precision Xtra; Abbott Diabetes Care Inc.) was used in this experiment according to the label descriptions of the manufacturer. The system consists of a handheld meter and electrochemical test strips. A $0.6-\mu \mathrm{L}$ blood sample is applied to the sensor after the test strip is inserted into the meter. Once the blood progresses through the test strip via capillary action, it reacts with glucose oxidase to form gluconic acid, which then reacts with the potassium ferricyanide to create potassium ferrocyanide, which reacts with the metal of the test strip electrodes, creating an electrical current. The current generated is directly proportional to the amount of glucose present in the blood. After $5 \mathrm{~s}$, the monitor displays the glucose concentration $(\mathrm{mmol} / \mathrm{L})$. The limits of quantification of the glucometer according to the test strip package insert are 1.1 to $27.8 \mathrm{mmol} / \mathrm{L}$.

This experiment included 81 peripartum Holstein dairy cows housed at the University of Guelph (Guelph, ON, Canada) dairy research herds. These animals were part of an observational study reported separately (Wittrock et al., 2012). Animals were cared for according to the Canadian Council on Animal Care guidelines (CCAC, 2009) and fed and managed conventionally. Data were collected on enrolled animals from $3 \mathrm{wk}$ before due date until 5 wk after calving between November 2010 and October 2011.

Duplicate blood samples $(\mathrm{n}=709)$ were taken from the coccygeal vein or artery into vacuum tubes (Vacutainer; Becton Dickson, Franklin Lakes, NJ) with the preservative sodium fluoride potassium oxalate $(\mathrm{NaF}$; the gold standard for glucose analysis, as NaF stops enzymatic activity of the glycolytic pathway; Peakman and Elliott, 2008) or without any preservative. After samples were obtained, the tubes were gently inverted 10 times to ensure thorough mixing with the preservative and placed in a chilled container. Glucometer readings were taken on the whole blood with no preservative immediately following collection. The glucose concentration $(\mathrm{mmol} / \mathrm{L})$ displayed on the glucometer was recorded. One meter was used for all of the glucose measurements. Blood was separated through centrifugation $(1,000 \times g$ for $30 \mathrm{~min})$. Serum was collected, frozen, and stored at $-20^{\circ} \mathrm{C}$ until submission to the Animal Health Laboratory (University of Guelph) for determination of glucose concentrations using a commercial reagent kit (GLUC3; Roche Diagnostics Corp.,
Indianapolis, IN) and auto-chemistry analyzer (Cobas c311; Roche Diagnostics Corp.). The analytical sensitivity of the glucose assay was $0.1 \mathrm{mmol} / \mathrm{L}$, and the inter- and intraassay coefficients of variation were 2.9 and $2.4 \%$, respectively.

Fifty-nine simplified GTT (Matteo et al., 2009) were conducted 1 wk before calving and consisted of an intravenous bolus infusion of $0.25 \mathrm{~g} / \mathrm{kg}$ dextrose (Dextrose 50\%; Vétoquinol Canada Inc., Lavaltrie, QC, Canada) based on standard estimated BW of $650 \mathrm{~kg}$. Duplicate blood samples were taken immediately before dextrose infusion and at 10 and 80 min after. Animals were classified as insulin resistant if the ratio of blood glucose concentration at $80 \mathrm{~min}$ after dextrose infusion to before infusion was $\geq 1.05$ (Matteo et al., 2009). We emphasize that this cut point is preliminary, under investigation, and requires validation.

Statistical analyses were performed using SAS (version 9.2; SAS Institute Inc., Cary, NC). Glucose concentrations obtained from the serum preserved with $\mathrm{NaF}$ and analyzed in the laboratory were considered the gold standard for all analyses. Samples from both routine data collection (at $-3,-2,-1,1,2,3,4$, and 5 wk relative to calving) and GTT were used, including 59 observations of circulating glucose at peak concentrations $10 \mathrm{~min}$ after the bolus infusion associated with the GTT. Pearson correlation coefficients were calculated. A Passing-Bablok linear regression correlation plot was performed, comparing the values from the glucometer to the gold standard laboratory measurements (Passing and Bablok, 1983). Using only correlation coefficients may not be suitable for evaluation of diagnostic test performance (Bland and Altman, 1986); therefore, a Bland-Altman plot was used to assess the residuals between the results from the Precision Xtra meter and the laboratory.

Using a GTT ratio of 1.05 (i.e., glucose concentration 80 min after infusion vs. just before infusion), the suitability of the Precision Xtra meter for assessing IR was assessed. The GTT ratios calculated from Precision Xtra glucose concentrations were compared with those calculated from the $\mathrm{NaF}$ samples analyzed in the laboratory. To identify an optimal test threshold for the glucometer relative to the proposed 1.05 laboratory result-based GTT ratio, glucometer GTT ratios were dichotomized. The optimal cut point was the value that produced the highest sum of sensitivity and specificity. Differences between GTT ratios measured by the glucometer and in the laboratory were evaluated using a paired $t$-test.

Figure 1 depicts a Passing-Bablok correlation plot between the glucose concentrations as measured by the laboratory and the glucometer. The Pearson correlation coefficient was $0.95(P<0.001)$. The intercept 


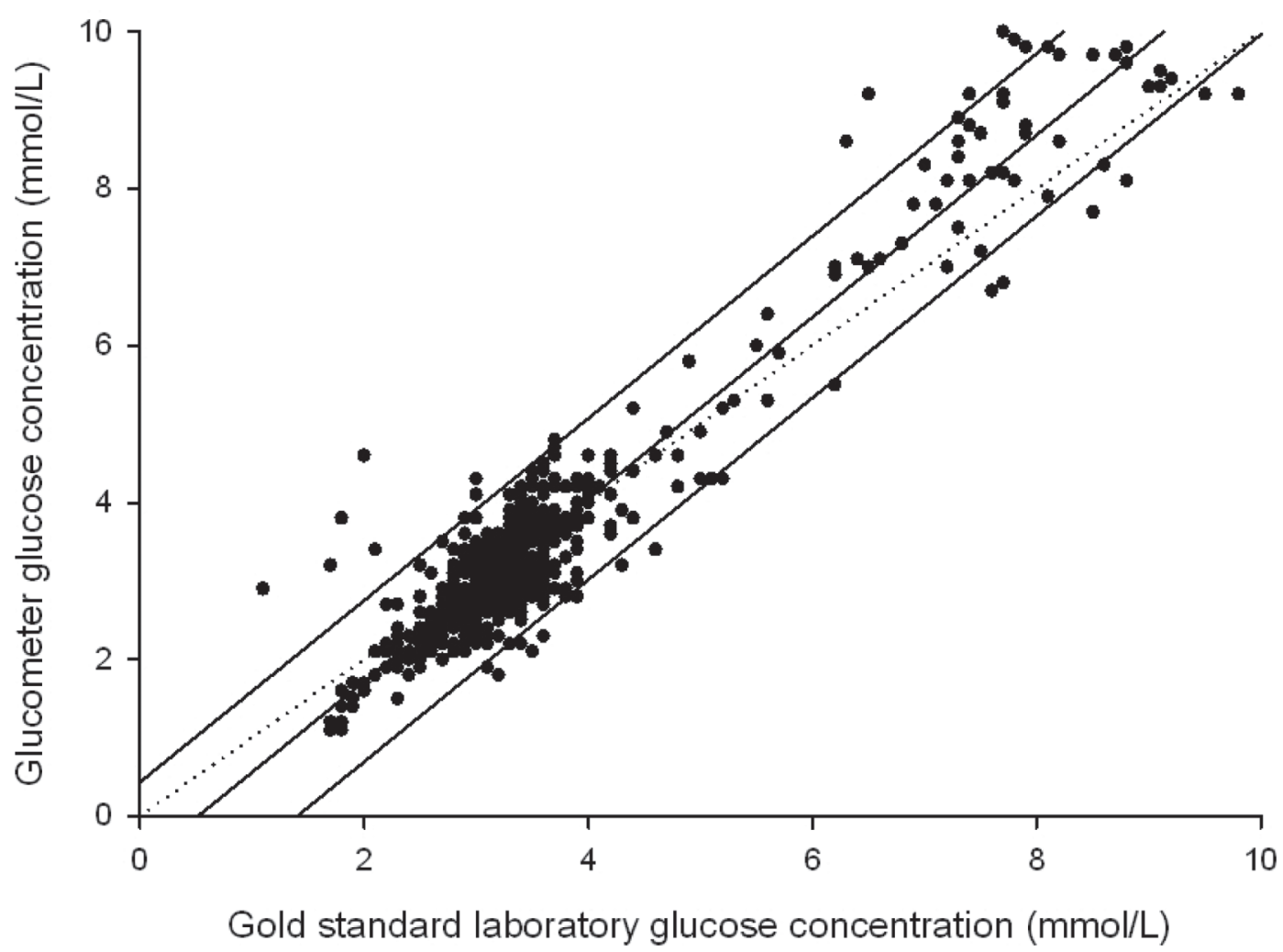

Figure 1. Passing-Bablok correlation plot for glucose concentrations from a glucometer (Precision Xtra; Abbott Diabetes Care Inc., Mississauga, ON, Canada) against serum preserved in sodium fluoride potassium oxalate and analyzed on an auto-chemistry analyzer (Cobas c311; Roche Diagnostics Corp., Indianapolis, IN). There were 709 samples from 81 cows, with the regression line (solid central black line), regression confidence limits (outer 2 solid black lines), and identity line $(\mathrm{x}=\mathrm{y}$; dotted line). Values above $6 \mathrm{mmol} / \mathrm{L}$ are peak concentrations observed $10 \mathrm{~min}$ after dextrose infusion during the glucose tolerance test (GTT), whereas those below $6 \mathrm{mmol} / \mathrm{L}$ occurred during routine sampling, before the GTT, or $80 \mathrm{~min}$ after the GTT intravenous dextrose infusion. Pearson correlation coefficient $=0.95(P<0.001)$.

differed from zero $(P<0.0001)$ and the slope was not equal to $1(P<0.0001)$, indicating some measurement error. Measurements in the physiologic range (2.3 to $5.2 \mathrm{mmol} / \mathrm{L}$; Quiroz-Rocha et al., 2009) were generally slightly lower with the glucometer than the laboratory value, whereas high glucose values were generally somewhat overestimated by the glucometer. We are not aware of a methodological reason for these differences. The relationship from linear regression analysis was as follows: laboratory glucose $=0.6+0.86 \times$ glucometer glucose. Plotting of the residuals between the glucometer and laboratory results against their mean revealed that the glucometer readings were, on average, 0.03 points lower than the laboratory results (Figure 2); overall, $96 \%$ of the observations fell between the $95 \%$ confidence intervals in the Bland-Altman plot (Figure 2 ), indicating good agreement. In the physiologic range $(<5.2 \mathrm{mmol} / \mathrm{L}), 98 \%$ of the observations fell within the $95 \%$ confidence interval, whereas greater discrepancy was evident with higher glucose values, where 20 of 65 $(31 \%)$ measurements with the glucometer were above the upper limit of the $95 \%$ confidence interval.
Using the 1.05 GTT ratio cut point proposed by Matteo et al. (2009), the ability of the glucometer to detect IR was compared with that of the laboratory glucose readings. The GTT ratio is based on measurements just before and $80 \mathrm{~min}$ after the bolus of dextrose, at which times glucose concentrations are almost all in the physiologic range, in which the accuracy of the glucometer was good and the GTT ratios from the glucometer and the laboratory were not different $(P=0.24)$. The sensitivity and specificity of the glucometer at predicting insulin sensitivity (based on the proposed GTT ratio of 1.05 ) were 84 and $52 \%$, respectively [kappa statistic (agreement beyond chance) $=0.37$. Values of kappa 0 to 0.20 indicate slight agreement; 0.21 to 0.40 , fair; 0.41 to 0.60 , moderate; 0.61 to 0.80 , substantial; and 0.81 to 1, almost perfect agreement (Dohoo et al., 2009). Given the differences in glucose concentration as measured by the hand-held meter, the sum of sensitivity and specificity was greatest at a cut point of 1.15 for the glucometer GTT ratio, producing a sensitivity of $69 \%$ and specificity of $85 \%$ (kappa $=0.53)$ relative to the 1.05 cut point for the laboratory measurements. 


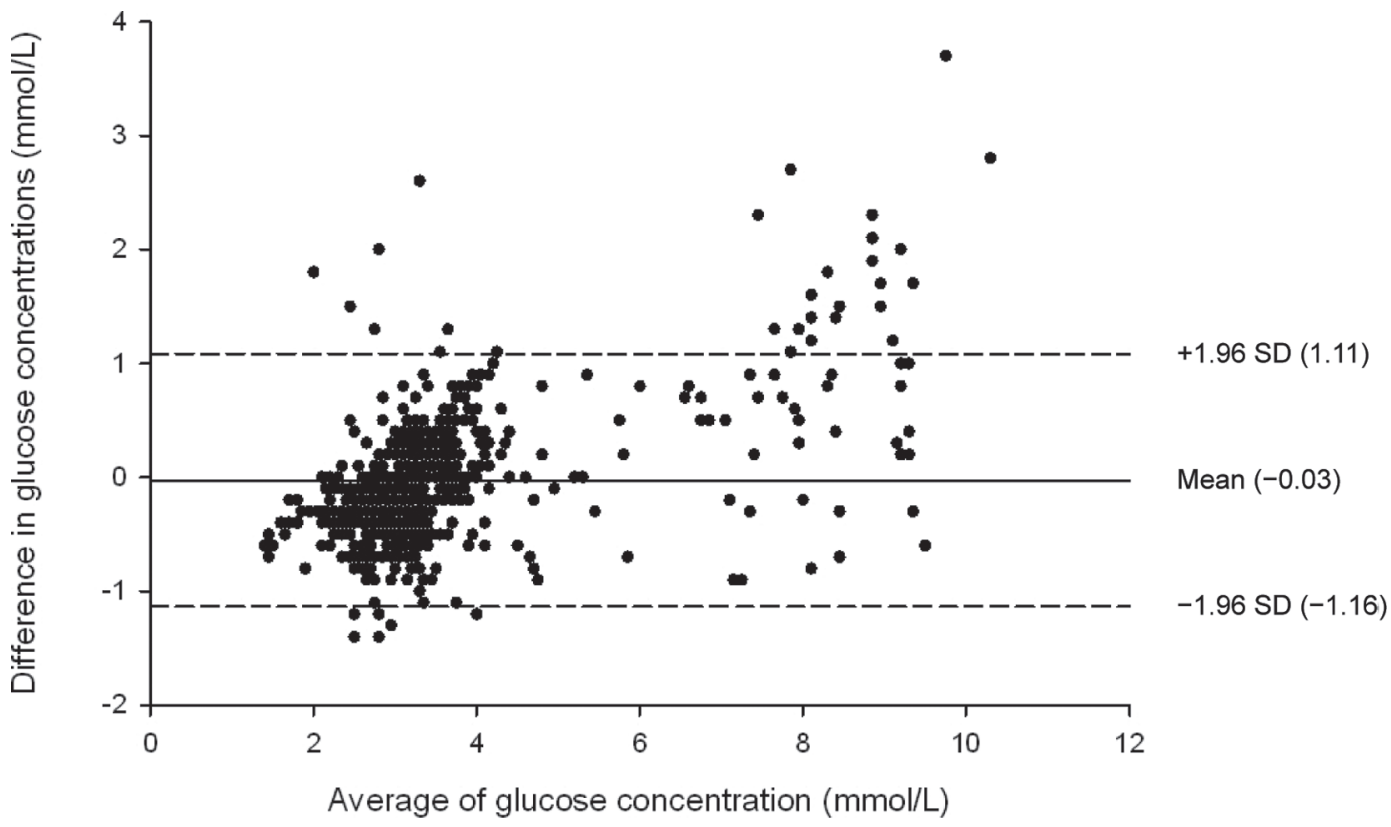

Figure 2. Bland-Altman plot ( $\mathrm{n}=709$ samples from 81 cows), with the mean glucose concentration as measured by the glucometer (Precision Xtra; Abbott Diabetes Care Inc., Mississauga, ON, Canada) and the serum preserved in sodium fluoride potassium oxalate and analyzed in a laboratory with a commercial reagent kit and auto-chemistry analyzer (Cobas c311; Roche Diagnostics Corp., Indianapolis, IN) on the x-axis, plotted against the difference in glucose concentrations determined by the glucometer and the laboratory on the y-axis. The mean difference is represented by the solid line and the $95 \%$ confidence limits by the dashed lines. Values above $6 \mathrm{mmol} / \mathrm{L}$ are peak concentrations observed 10 min after dextrose infusion during the glucose tolerance test (GTT); those below $6 \mathrm{mmol} / \mathrm{L}$ occurred during routine sampling, before the GTT, or 80 min after the GTT intravenous dextrose infusion.

Although the Precision Xtra meter has been validated for use in dairy cows for measuring subclinical ketosis (Iwersen et al., 2009), this is the first study to validate its ability to determine glucose concentrations in dairy cows. This hand-held glucometer performed well compared with the laboratory. The correlation between the gold standard and glucometer was high, and the evaluation of the residuals revealed an even distribution, with most observations falling within the $95 \%$ confidence intervals, especially for values within the normal range for dairy cows (i.e., not samples taken at peak glucose concentrations $10 \mathrm{~min}$ after GTT dextrose infusion). These results suggest that the Precision Xtra meter may be a useful point-of-care tool. Other glucometers have been assessed for performance in dairy cows (Galvão et al., 2010); however, there may be practical benefits in the ability to use one instrument to measure both glucose and ketones in dairy cows.

More investigation is required to determine whether the simplified GTT is valid and useful for identifying animals at risk of developing disease, as the current research is limited. Due to the strong correlation and low variation in residuals between glucose concentrations obtained from whole blood by the Precision Xtra meter and plasma glucose concentrations, the hand-held glucometer appears suitable for rapid measurement of glucose under field conditions in dairy cattle.

\section{REFERENCES}

Bland, J. M., and D. G. Altman. 1986. Statistical methods for assessing agreement between two methods of clinical measurement. Lancet 1:307-310.

CCAC (Canadian Council on Animal Care). 2009. CCAC guidelines on: The Care and Use of Farm Animals in Research, Teaching and Testing. CCAC, Ottawa, ON, Canada.

Dohoo, I. R., W. Martin, and H. Stryhn. 2009. Veterinary Epidemiologic Research. 2nd ed. VER Inc., Charlottetown, PEI, Canada.

Fall, N., Y. T. Gröhn, K. Forslund, B. Essen-Gustafsson, R. Niskanen, and U. Emanuelson. 2008. An observational study on early-lactation metabolic profiles in Swedish organically and conventionally managed dairy cows. J. Dairy Sci. 91:3983-3992.

Galvão, K. N., M. J. B. F. Flaminio, S. B. Brittin, R. Sper, M. Fraga, L. Caixeta, A. Ricci, C. L. Guard, W. R. Butler, and R. O. Gilbert. 2010. Association between uterine disease and indicators of neutrophil and systemic energy status in lactating Holstein cows. J. Dairy Sci. 93:2926-2937.

Garverick, H. A., M. N. Harris, R. Vogel-Bluel, J. D. Sampson, J. Bader, W. R. Lamberson, J. N. Spain, M. C. Lucy, and R. S. Youngquist. 2013. Concentrations of nonesterified fatty acids and glucose in blood of periparturient dairy cows are indicative of pregnancy success at first insemination. J. Dairy Sci. 96:181-188.

Hayirli, A. 2006. The role of exogenous insulin in the complex of hepatic lipidosis and ketosis associated with insulin resistance phenomenon in postpartum dairy cattle. Vet. Res. Commun. 30:749-774.

Iwersen, M., U. Falkenberg, R. Voigtsberger, D. Forderung, and W. Heuwieser. 2009. Evaluation of an electronic cowside test to detect subclinical ketosis in dairy cows. J. Dairy Sci. 92:2618-2624.

Kahn, C. R. 1978. Insulin resistance, insulin sensitivity, and insulin unresponsiveness: A necessary distinction. Metabolism 27:18931902.

Leury, B. J., L. H. Baumgard, S. S. Block, N. Segoale, R. A. Ehrhardt, R. P. Rhoads, D. E. Bauman, A. W. Bell, and Y. R. Boisclair. 2003. Effect of insulin and growth hormone on plasma leptin in 
periparturient dairy cows. Am. J. Physiol. Regul. Integr. Comp. Physiol. 285:R1107-R1115.

Lozner, E. L., A. W. Winkler, F. H. L. Taylor, and J. P. Peters. 1941. The intravenous glucose tolerance test. J. Clin. Invest. 20:507515.

Matteo, G., C. Chiara, C. Mauro, and M. Massimo. 2009. Cows response to glucose tolerance test (GTT) and periparturient diseases: Preliminary study. J. Dairy. Sci. 92(E-Suppl. 1):385. (Abstr.)

Oetzel G. and S. McGuirk. 2008. Study of blood glucose in a dairy herd with high incidence of ketosis and hand-held meter for cowside evaluation of blood beta-hydroxybutyrate. Page 234 in Proc. 41st Conf. Am. Bovine Pract. Assoc., Charlotte, NC. Am. Assoc. Bovine Pract., Auburn, AL. (Abstr.)

Passing, H., and W. Bablok. 1983. A new biometrical procedure for testing the equality of measurements from two different analytical methods: Application of linear regression procedures for method comparison studies in clinical chemistry, part I. J. Clin. Chem. Clin. Biochem. 21:709-720.
Peakman, T. C., and P. Elliott. 2008. The UK Biobank sample handling and storage validation studies. Int. J. Epidemiol. 37:i2-i6.

Quiroz-Rocha, G. F., S. J. LeBlanc, T. F. Duffield, D. Wood, K. E. Leslie, and R. M. Jacobs. 2009. Reference limits for biochemical and hematological analytes of dairy cows one week before and one week after parturition. Can. Vet. J. 50:383-388.

Riuzzi, S., E. Fiore, M. Gianesella, and M. Morgante. 2012. Correlation between a field glucose tolerance test (fgtt) in the transition dairy cow and b-ohb, glycemia and disease incidence detected in a field trial. Page 96 in Proc. 27th World Buiatrics Congress, Lisbon, Portugal. World Assoc. for Buiatrics. (Abstr.)

Schoenberg, K. M., and T. R. Overton. 2010. The changing roles of insulin during the transition period. Pages $175-185$ in Proc. Cornell Nutrition Conf., East Syracuse, NY. Cornell Univ., Ithaca, NY. (Abstr.)

Wittrock, J. A. M., T. F. Duffield, D. Bienzle, and S. J. LeBlanc. 2012 Neutrophil function in the transition period and reproductive tract health in dairy cows. J. Dairy Sci. 95(Suppl. 2):233. (Abstr.) 